\title{
El flujo como indicador del efecto terapéutico del ácido folínico en la isquemia-reperfusión
}

\author{
J. San Cristóbal, I. Cearra, B. Otero, T. Martínez-Astorquiza, H. Marín e I. García-Alonso \\ Laboratorio de Cirugía Experimental. Facultad de Medicina y Odontología. Universidad del País Vasco. Vizcaya
}

\section{RESUMEN}

Objetivo: estudio sobre reperfusión intestinal con una doble finalidad: a) valorar la utilidad de la medición del flujo capilar intestinal mediante láser-doppler para el estudio de la reperfusión intestinal; y b) comparar el efecto de las formas racémica y levo del ácido folínico en su tratamiento.

Diseño experimental: modelo murino de isquemia intestinal mediante clampaje completo de la arteria mesentérica superior durante 90 minutos. Se compara la evolución de tres grupos de tratamiento: suero salino, ácido folínico y ácido levofolínico. Se analiza la evolución de parámetros bioquímicos (niveles de creatín kinasa, láctico deshidrogenasa y fosfatasa alcalina a los 60 minutos y dos y siete días de restablecer el flujo sanguíneo), flujo capilar en yeyuno e íleon mediante láser-doppler (durante isquemia y tras primera hora de reperfusión), lesión mucosa intestinal, y curva de supervivencia.

Resultados: el láser-doppler permitió analizar con fiabilidad el efecto de los tratamientos sobre el flujo capilar durante reperfusión intestinal. El ácido levofolínico mejoró el flujo capilar en el íleon a partir de los 25 minutos de reperfusión, a la vez que disminuyó la lesión mucosa en el mismo tramo intestinal el séptimo día de evolución $(\mathrm{p}<0,05)$. En cambio, produjo un aumento inicial de los enzimas séricos durante la reperfusión, y no modificó la supervivencia.

Conclusiones: los cambios en la microcirculación valorados con láser-doppler han mostrado cierta relación con las variaciones observadas en los estudios anatomopatológicos. No se ha podido demostrar que la forma levo del ácido folínico ejerza mayor protección que el compuesto racémico.

\begin{abstract}
Objective: an intestinal reperfusion study with two aims: a) to assess the usefulness of intestinal capillary blood flow measurement by laser-Doppler for intestinal reperfusion studies; and b) to compare the effects of racemic and levo forms of folinic acid in treating the syndrome.

Experimental design: a murine model of intestinal ischemia by completely clamping the superior mesenteric artery for 90 minutes. A comparison was made of three treatment groups: saline, folinic acid, and levo-folinic acid. The following factors were analyzed: changes in biochemical parameters (levels of creatine kinase, lactic dehydrogenase, and alkaline phosphatase at 60 minutes, and at two and seven days after restoring blood flow), capillary flow in the jejunum and ileum by laser-Doppler (during ischemia and after the first hour of reperfusion), intestinal mucosa injury, and survival curve.

Results: laser-Doppler provided reliable data on how the different treatments affected capillary flow during intestinal reperfusion. Levo-folinic acid improved capillary flow in the ileum after 25 minutes of reperfusion, and also reduced mucosal injury in the same stretch of intestine by the seventh day post-reperfusion $(\mathrm{p}<$ $0.05)$. On the other hand, it produced an initial increase in serum enzymes during reperfusion, and did not modify survival.

Conclusions: the changes observed in intestinal capillary blood flow measurement by laser-Doppler have similarities with the effects of drugs on pathological mucosal changes. We could not prove that the levo form of folinic acid has a stronger protective effect versus racemic folinic acid in intestinal ischemia-reperfusion syndrome.
\end{abstract}

Key words: Ischemia-reperfusion. Intestine. Folinic acid. LaserDoppler. Capillary blood flow.
Palabras clave: Isquemia-reperfusión. Intestino. Ácido folínico. Láser-doppler. Flujo capilar.

San Cristóbal J, Cearra I, Otero B, Martínez-Astorquiza T, Marín H, García-Alonso I. El flujo capilar como indicador del efecto terapéutico del ácido folínico en la isquemia-repefusión. Rev Esp Enferm Dig 2007; 99: 25-32.

Recibido: 01-02-06.

Aceptado: 06-10-06.

Correspondencia: Ignacio García-Alonso. Laboratorio de Cirugía Experimental. Facultad de Medicina y Odontología. Universidad del País Vasco. Barrio Sarriena, s/n. 48940 Leioa. Vizcaya. e-mail: ignacio.galonso@ehu.es

\section{INTRODUCCIÓN}

El síndrome de isquemia-reperfusión, especialmente a nivel del intestino, ha sido objeto de numerosos estudios durante las últimas décadas. Sin embargo, todavía hoy 
día sigue presentando aspectos poco conocidos y las pautas terapéuticas ensayadas necesitan seguir mejorándose. Desde que McCord implicara a los radicales libres del oxígeno en la fisiopatología de este proceso (1), se han ido descubriendo nuevos mediadores del daño celular a nivel del intestino (2-6). En la actualidad se acepta que los leucocitos polimorfonucleares son los principales agentes del daño por reperfusión, debido a las lesiones que provocan en los endotelios vasculares (7-9). Esta lesión endotelial agrava el daño histológico en el tejido reperfundido y extiende las lesiones a otros órganos (riñón, hígado, pulmón). Por este motivo, el estudio de la microcirculación durante la reperfusión de un órgano isquémico mediante láser-doppler (10) puede constituir una herramienta muy útil para evaluar las medidas terapéuticas. Para ello, resulta necesario compararlo con los otros parámetros utilizados hasta ahora: alteraciones anatomopatológicas (11), modificaciones de los enzimas séricos (12), y supervivencia de los animales sometidos a procesos de isquemia-reperfusión $(13,14)$.

Por otra parte, y dado que los radicales libres del oxígeno parecen ser los iniciadores de este proceso patológico, se ha estudiado la capacidad de diversos fármacos antioxidantes para reducir el daño provocado por la reperfusión. Uno de los agentes más eficaces ensayados, ha sido el ácido fólico en su forma activa (ácido folínico). En nuestra experiencia, la administración intravenosa de ácido folínico durante los minutos previos al restablecimiento del flujo mesentérico tras el bloqueo completo de la arteria mesentérica superior en la rata disminuye tanto la gravedad de las lesiones mucosas (15), como la mortalidad (16). En la actualidad, se dispone de un preparado que contiene exclusivamente el enantiómero levo del ácido folínico (levofolínico), que es la forma biológicamente activa. Sin embargo, no existen datos acerca de si esta nueva presentación farmacológica resulta más eficaz en el control de las lesiones por reperfusión que la mezcla racémica ensayada anteriormente.

El presente trabajo se ha diseñado para estudiar la eficacia de la monitorización del flujo capilar por láser-doppler como nueva herramienta de estudio de la reperfusión de tejidos isquémicos. Así, las valoraciones realizadas a partir del registro de flujos capilares se comparan con los datos aportados por la anatomía patológica, la bioquímica sérica y los estudios de supervivencia. Al mismo tiempo, se emplean estos cuatro parámetros para comparar la utilidad terapéutica de la forma levo del ácido folínico frente a la mezcla racémica de dicho compuesto, en un modelo de isquemia-reperfusión intestinal en la rata.

\section{MÉTODOS}

\section{Animales y procedimientos quirúrgicos}

El trabajo se ha realizado con ratas WAG/RijCrl, machos, de 12 semanas de edad. Los animales se han esta- bulado en jaulas individuales con libre acceso al agua y comida (Panlab A-04), con un ciclo constante de luz-oscuridad de 12 horas. En todo momento se ha respetado lo establecido en la "Guía nacional española para el cuidado del animal en experimentación y otros fines científicos" (Real Decreto 223/88).

Bajo anestesia con $\mathrm{Nembutal}^{\circledR}(40 \mathrm{mg} / \mathrm{kg}$ i.p.) se practicó una laparotomía media y se disecó la arteria mesentérica superior en su nacimiento, a su salida de la aorta. La isquemia mesentérica se indujo mediante la colocación de dos clips de Yasargil durante 90 minutos. Diez minutos antes de restablecer el flujo mesentérico se puncionó la vena cava inferior suprarrenal (aguja 27G), administrando los diferentes fármacos disueltos en $2 \mathrm{ml}$ de suero salino. La velocidad de infusión $(0,2 \mathrm{ml} / \mathrm{min})$ se controló mediante una bomba de émbolo (Cordis Hyperion $^{\circledast}$ ). Completado el tiempo de isquemia se retiraron los clips y se comprobó el restablecimiento del latido en las arterias rectas del mesenterio.

\section{Grupos y series experimentales}

Se han realizado tres experiencias (Tabla I), utilizando en cada una de ellas tres grupos de 10 animales. El grupo 1, que sirvió como control, recibió únicamente suero salino; el grupo 2 fue tratado con ácido folínico (Lederlefolín ${ }^{\circledast} 2,5$ $\mathrm{mg} / \mathrm{kg}$ ); y el grupo 3 recibió ácido levofolínico (Isovorín ${ }^{\circledast}$ $2,5 \mathrm{mg} / \mathrm{kg}$ ).

En la primera experiencia (serie I) los animales fueron sometidos al proceso de isquemia-reperfusión, y se les administraron los distintos tratamientos. Transcurridas 48 horas se procedió a sacrificar los animales para estudiar la lesión mucosa y los enzimas séricos.

En la segunda experiencia (serie II) se estudió el restablecimiento del flujo capilar en el intestino mediante tres sondas apoyadas suavemente sobre el borde antimesentérico de la serosa del yeyuno, del íleon y de un tramo intermedio del intestino delgado, conectadas a un fluxómetro láser-doppler (Oxford Optronix ${ }^{\circledast}$ ). Para conocer el valor del flujo capilar en condiciones basales y en situación de isquemia se han registrado de modo continuo, durante al menos dos minutos de medición estable, los valores de flujo capilar antes de colocar los clips (medida basal) y tras la inducción de la isquemia. Durante la reperfusión se ha

Tabla I. Series experimentales

\begin{tabular}{ccc}
\hline & Momento del sacrificio & Estudios realizados \\
\hline Serie I & Día 2 (48 h) & Daño (enzimas y daño mucoso) \\
$48 \mathrm{~h}$
\end{tabular}


mantenido un registro continuo, calculándose posteriormente el valor medio de flujo durante tres intervalos de diez minutos: inicial ( $0^{\prime}$ a $10^{\prime}$ ), intermedio (25' a $35^{\prime}$ ) y final (50' a 60'). A partir de estos datos se ha calculado el porcentaje de recuperación de flujo capilar con respecto al valor basal en cada animal (Tabla II). Terminada la medición se procedió al sacrificio de los animales.

La tercera experiencia (serie III) se orientó a estudiar la supervivencia. Para ello, los animales fueron sometidos a los mismos procedimientos que en la primera serie, realizándose un seguimiento de los fallecimientos: horario durante las primeras 12 horas, y diario hasta el día séptimo. En ese momento se sacrificaron los animales supervivientes.

\section{Parámetros estudiados}

Al sacrificar los animales se obtuvieron tres muestras consecutivas de intestino de $1 \mathrm{~cm}$ de longitud, tanto del íleon terminal como del yeyuno proximal, las cuales se incluyeron en parafina. En secciones histológicas de $6 \mathrm{~mm}$ teñidas con hematoxilina-eosina se valoró el grado de lesión en cada uno de los cuatro cuadrantes de cada muestra, de acuerdo con la escala modificada de Park (11) (Tabla III).

Para estudiar los enzimas séricos, se extrajeron $2 \mathrm{ml}$ de sangre en el momento del sacrificio. Además, para disponer de sueros control, en la segunda experiencia se practicó otra extracción, previa a la colocación de los clips, tras la cual se administraron $2 \mathrm{ml}$ de suero salino. Las determinaciones enzimáticas ( $\mathrm{LDH}$, fosfatasa alcalina y crea-

\section{Tabla II. Cálculo del porcentaje de recuperación del flujo} capilar en el intestino

$$
\% \text { de recuperación }=\frac{\text { Dato }- \text { Isquemia }}{\text { Basal }- \text { Isquemia }} \times 100
$$

- Dato: flujo medio registrado en cada intervalo (inicial, intermedio y final) de la reperfusión

- Isquemia: flujo durante la isquemia

- Basal: flujo antes de la isquemia

Tabla III. Escala de daño histológico (adaptada de Parks y cols.) (11)

0. Sin daño aparente

1. Despegamiento apical de la mucosa en las vellosidades

2. Denudación apical de las vellosidades $(<20 \%)$

3. Pérdida parcial del epitelio de la vellosidad (del 20-50\%)

4. Pérdida importante del epitelio de la vellosidad (50-80\%)

5. Pérdida total del epitelio de la vellosidad (> 80\%)

6. Aplanamiento de las vellosidades

7. Destrucción de las vellosidades tinkinasa) se realizaron en un espectrofotómetro (Schimazu $^{\circledR}$.

El estudio estadístico se llevó acabo con el programa $\mathrm{GraphPad}^{\circledR}$. Los resultados se han expresado como medias y desviación estándar. La supervivencia se ha estudiado mediante curvas de Kaplan-Meier, realizándose las comparaciones entre ellas mediante el test de Logrank. Las comparaciones entre los resultados de los tres grupos experimentales se realizaron mediante análisis de la varianza, aplicándose el test de Newman-Keuls en aquellos casos en los que el ANOVA mostró un $\mathrm{p}<0,05$.

\section{RESULTADOS}

\section{Estudios de supervivencia}

La mortalidad debida al síndrome de isquemia-reperfusión se manifestó en todos los casos dentro de las primeras 24 horas. En nuestra experiencia, la supervivencia en el grupo control se situó en el $70 \%$. Ninguno de los dos tratamientos ensayados indujo modificaciones significativas en este parámetro (Fig. 1). De hecho, aunque la supervivencia entre los animales tratados con levofolínico se situó en el $50 \%$, esta diferencia no fue significativa $(\mathrm{p}=0,58)$.

\section{Evolución del restablecimiento del flujo capilar}

En el íleon de los animales no tratados, tras retirar el clamp de la arteria mesentérica superior, el flujo capilar alcanzó aproximadamente un $20 \%$ del valor basal. Durante los sesenta minutos siguientes el flujo capilar disminuyó paulatinamente hasta situarse en torno al $15 \%$ (Fig. 2).

En los animales tratados con folínico, los valores de flujo en los primeros momentos de la reperfusión se si-

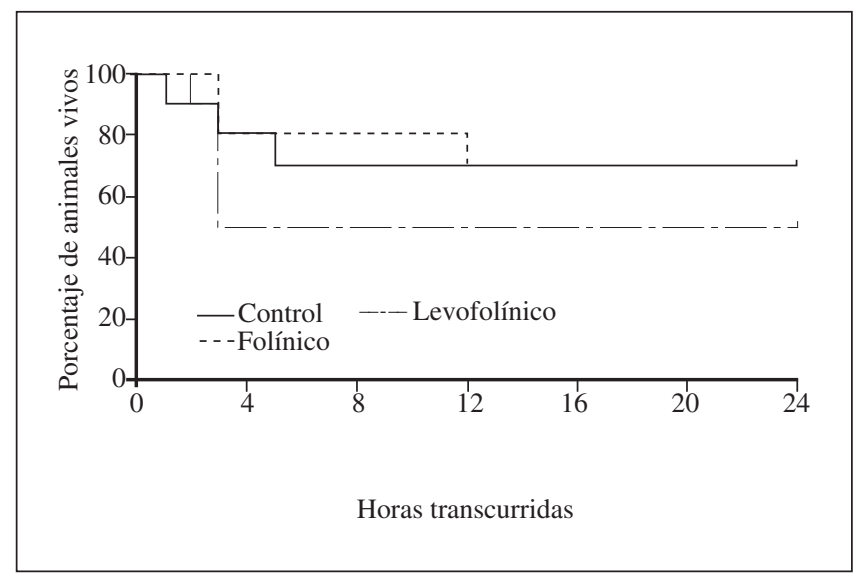

Fig. 1. Curva de supervivencia en los tres grupos experimentales. La diferencia frente a los tratados con levofolínico no alcanza significación estadística. 

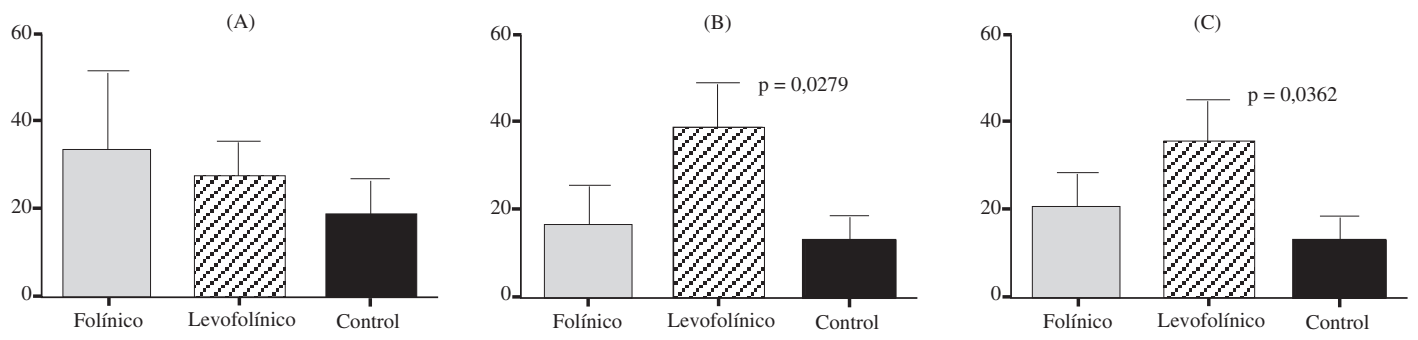

Fig. 2. Flujo capilar durante la reperfusión del íleon: A $0^{\prime}-10^{\prime}$, B $25^{\prime}-35^{\prime}$, C 50'-60'. Los resultados se expresan como porcentaje respecto al flujo basal. Los valores de la p se refieren a la comparación frente al grupo control.

tuaron en torno al 35\%, sin que esta diferencia alcanzara significación estadística. A lo largo de la primera hora de reperfusión el flujo disminuyó ligeramente, aunque manteniéndose siempre por encima de las cifras del grupo control, pero sin alcanzar significación estadística.

En cambio, los animales tratados con levofolínico mostraron un comportamiento diferente en la evolución de su flujo capilar a nivel del íleon. Si bien las cifras iniciales fueron similares a las del grupo de folínico (Fig. $3 \mathrm{~A}$ ), a lo largo de la reperfusión aumentaron hasta situarse en torno a un $50 \%$ del valor basal (intervalo intermedio $\mathrm{p}=0,0279$; final $\mathrm{p}=0,0362$ ).

El comportamiento del flujo capilar durante la reperfusión del yeyuno fue bastante similar a lo observado en el íleon. Sin embargo, a este nivel las diferencias no alcanzaron significación estadística en ningún momento del estudio (Fig. 3).

\section{Valoración del daño de la mucosa intestinal}

En las muestras obtenidas al final de la reperfusión se objetivó un importante grado de lesión, que, valorado con la escala recogida en la tabla II, se situó en torno al 5 para el yeyuno y en torno al 6 para el íleon. Ninguno de los dos tratamientos logró mejorías significativas. En las muestras obtenidas dos días después de la agresión isquémica se apreció una importante disminución del grado lesional en todos los grupos $(\mathrm{p}<0,01)$, que se situó en todos los casos por debajo de 3 (Fig. 4), sin que de nuevo hubiera diferencias atribuibles a ninguno de los dos fármacos, a la vez que tampoco existieron diferencias entre yeyuno e íleon. Los estudios realizados siete días después de la reperfusión mostraron un nivel de daño histológico similar al observado a las 48 horas. La única excepción la encontramos en el íleon de los animales tratados con levofolínico, donde se produjo una mejoría significativa frente a lo observado a las 48 horas $(2,6$ vs. $1,5, \mathrm{p}<0,05)$.

\section{Modificación de los enzimas séricos}

\section{Lactato deshidrogenasa}

En nuestros animales, las extracciones realizadas antes de iniciar la isquemia mostraron concentraciones séricas de LDH en torno a las $300 \mathrm{UI} / \mathrm{l}$. En cambio, las muestras obtenidas tras una hora de reperfusión alcanzaron valores en torno a $750 \mathrm{UI} / 1$, tanto en los animales tratados como en los controles $(\mathrm{p}<0,01)$. El siguiente registro, realizado a las 48 horas, mostró ya valores normales tanto en los controles como en los animales tratados con folínico. Sin embargo, los animales tratados con levofolínico presentaron valores significativamente elevados todavía en las $48 \mathrm{~h}$ ( 700 UI/l, p < 0,05). Por fin, los análisis realizados el $7^{\circ}$ día mostraron valores normales en todos los animales (Fig. 5).

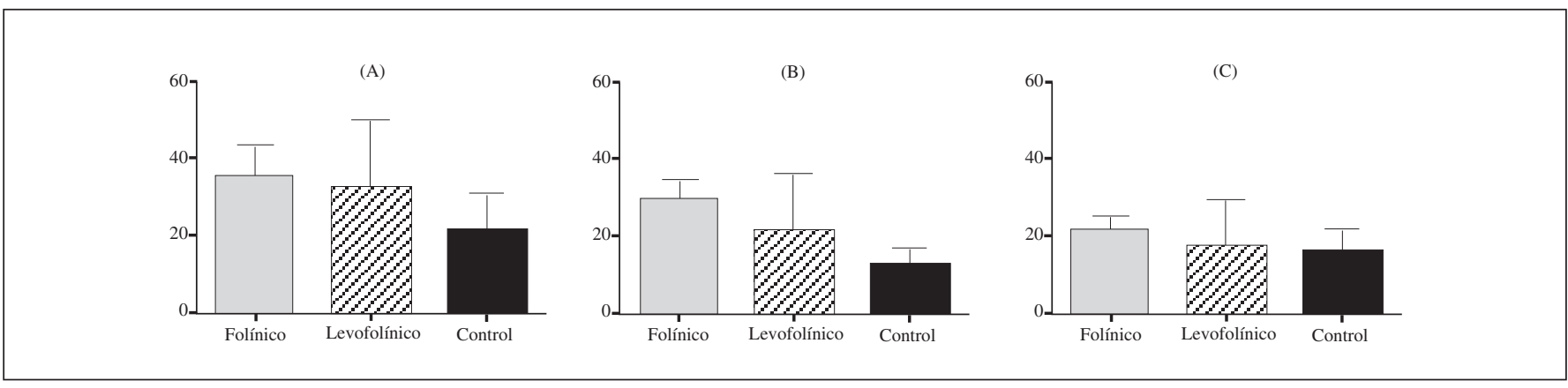

Fig. 3. Flujo capilar durante la reperfusión del yeyuno: A 0'-10', B 25'-35', C 50'-60'. Los resultados se expresan como porcentaje respecto al flujo basal. Los valores de la p se refieren a la comparación frente al grupo control. 


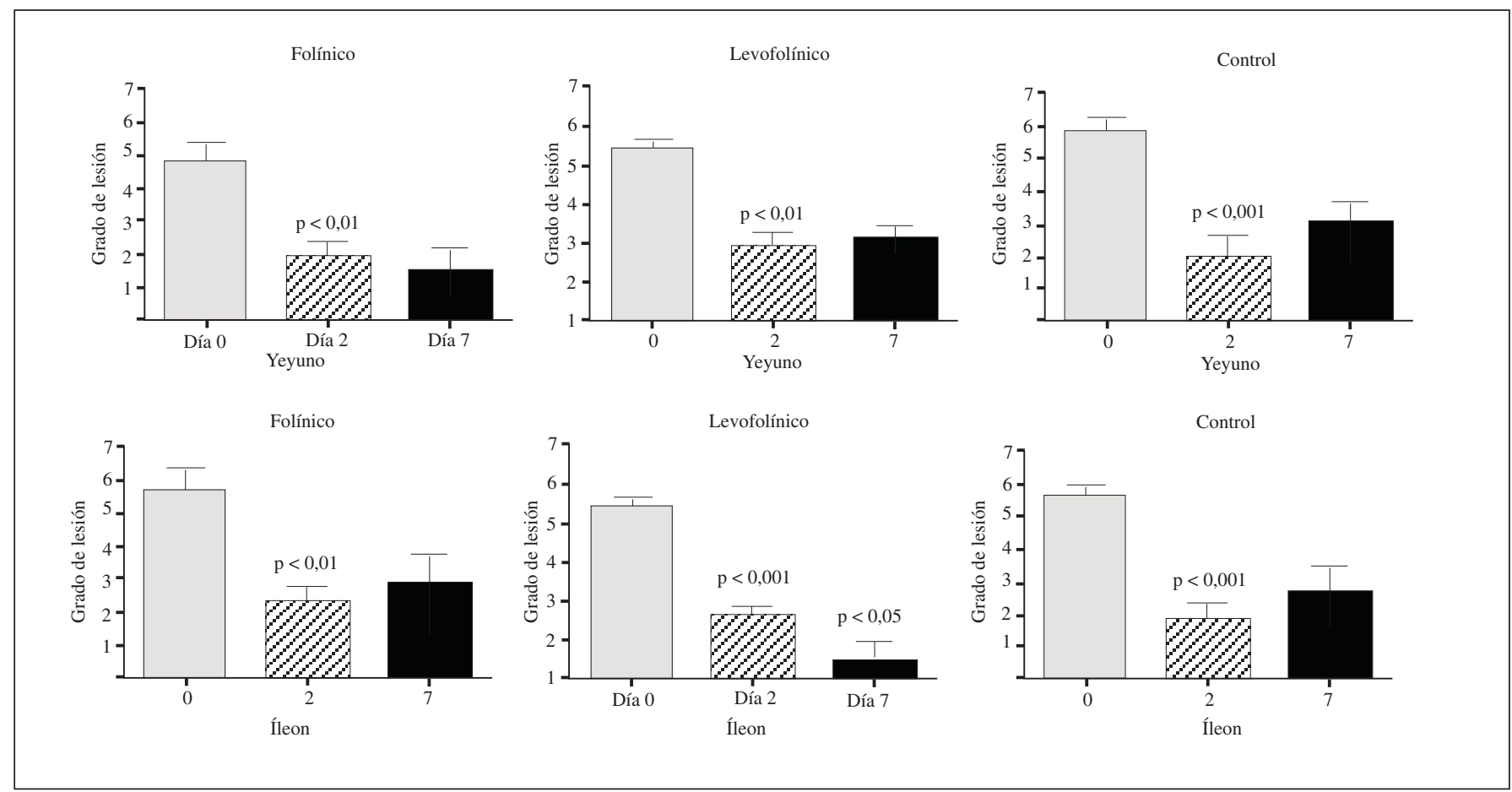

Fig. 4. Daño mucoso en yeyuno e íleon valorado tras una hora de reperfusión y en los días 2 y 7 tras la agresión isquémica (las comparaciones se realizan con la medición anterior -columna de la izquierda-).

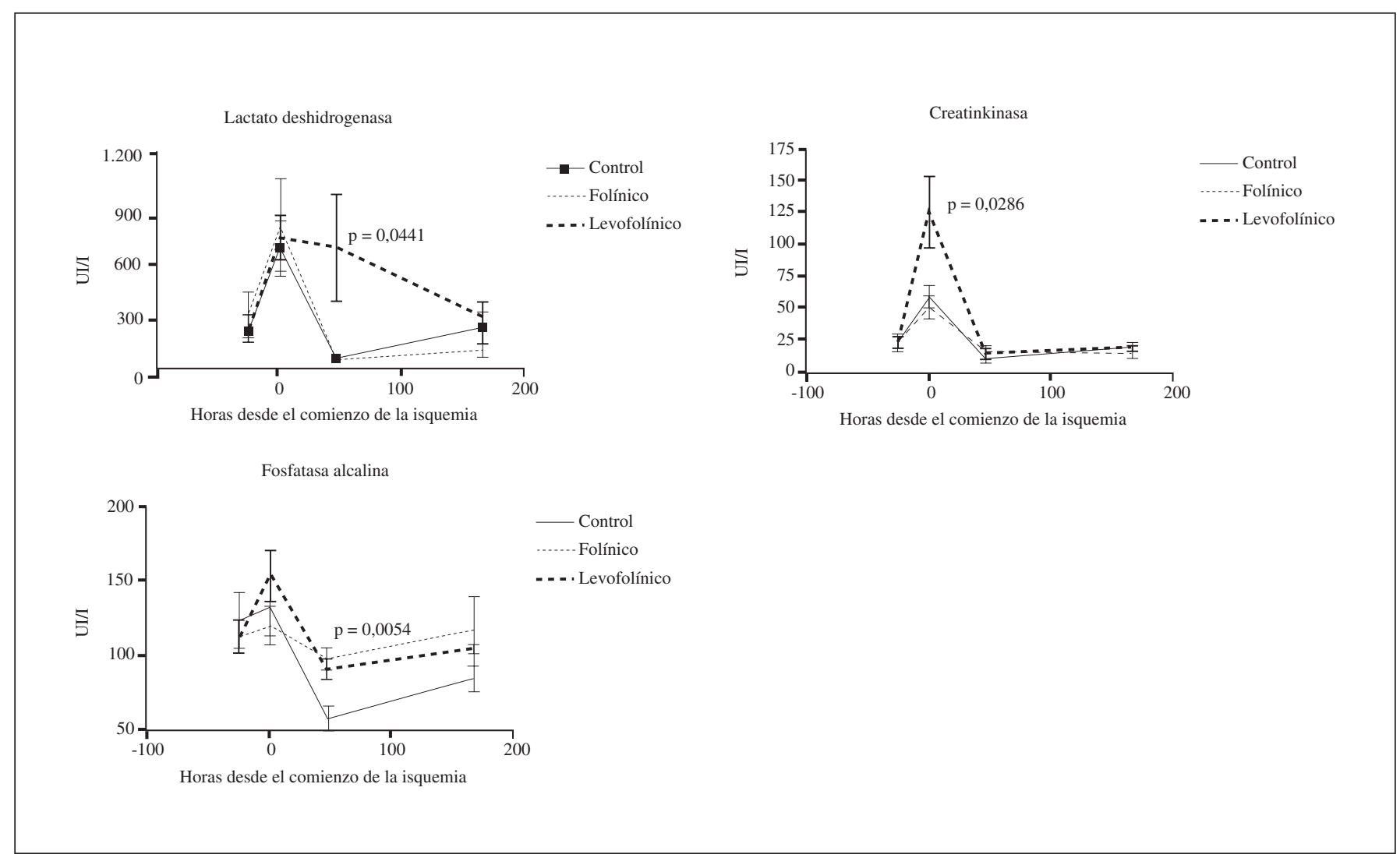

Fig. 5. Evolución de los valores séricos de las enzimas estudiadas. Los valores de la p se refieren a la comparación frente al grupo control en ese momento del estudio. 


\section{Fosfatasa alcalina}

Los valores séricos de fosfatasa alcalina al final de la primera hora de reperfusión se mantuvieron dentro de la normalidad en todos los grupos experimentales. Tras 48 horas, en el grupo control se observó una disminución drástica de dichos valores $(\mathrm{p}<0,01)$, recuperándose la normalidad en el séptimo día. En cambio, los animales tratados con tanto folínico como con levofolínico no mostraron modificación alguna (Fig. 5).

\section{Creatinkinasa}

Al finalizar la primera hora de reperfusión se encontró en los tres grupos experimentales un aumento importante de los valores séricos de CK $(\mathrm{p}<0,05)$; sin embargo, en los animales tratados con levofolínico el incremento fue mucho más acusado $(124 \pm 68 \mathrm{UI} / \mathrm{l} ; \mathrm{p}<$ $0,05)$ que en los tratados con folínico $(50 \pm 22$ UI/l $)$ o el grupo control $(59 \pm 23 \mathrm{UI} / \mathrm{l})$. Los estudios realizados a las 48 horas y el séptimo día mostraron valores normales en todos los casos.

\section{DISCUSIÓN}

¿Permite la medición de microflujos mediante láserdoppler valorar el efecto terapéutico de los tratamientos aplicados en un modelo de isquemia-reperfusión intestinal? Esta era la primera pregunta a contestar con nuestro trabajo. En este sentido, nuestras mediciones con láser-doppler han puesto en evidencia un efecto positivo del levofolínico limitado al íleon. Al mismo tiempo, el estudio anatomopatológico sólo encuentra efecto positivo en el íleon de aquellos animales tratados con dicho fármaco (analizado en el séptimo día de evolución). Podría decirse por tanto, que el láser-doppler muestra una sensibilidad similar a la de la anatomía patológica, pero de manera más precoz. Por otra parte, la falta de resultados positivos del análisis de la supervivencia parece privar a nuestro trabajo de una de las comparaciones previstas. Si bien esto es cierto, también lo es que el hecho de que el láser-doppler ha permitido detectar un efecto farmacológico que hubiera escapado al análisis de supervivencia.

No hemos encontrado publicaciones sobre el efecto del ácido folínico sobre la supervivencia tras isquemia intestinal. Tan sólo disponemos de los resultados previos de nuestro grupo en dos modelos muy similares al actual; en ambos el fármaco utilizado ha sido el Lederlefolín ${ }^{\circledast}$ (mezcla racémica del ácido folínico). En uno de ellos (15), que utilizó hembras Sprague-Dawley de 200 g, el ácido folínico disminuyó la mortalidad tras 120 minutos de isquemia (56 vs. $25 \%, \mathrm{p}<0,05)$. En el otro (17), a pesar de reducirse el tiempo de isquemia a 60 minutos, la mortalidad propia del modelo fue más elevada, debido a que las técnicas de monitorización hemodinámica utilizadas aumentaron la agresividad del experimento. En este modelo, el ácido folínico redujo la mortalidad desde el 83 hasta un $50 \%$, aunque dicha reducción no alcanzó significación estadística.

En cambio, en el presente trabajo, con un tiempo de isquemia intermedio (90 minutos), la mortalidad ha sido ligeramente superior al modelo de 2 horas de isquemia; esto puede deberse de nuevo a la mayor agresividad de la cirugía, ya que la laparotomía permaneció abierta durante toda la experiencia para permitir una monitorización constante del flujo intestinal. Sin embargo, al igual que ocurrió con el segundo trabajo citado (17), en esta ocasión tampoco la reducción de la mortalidad aportada por el folínico ha alcanzado significación estadística (70 vs. $50 \%$ ).

A la vista de estos datos, resulta todavía más sorprendente el hecho de que los animales tratados con levofolínico hayan presentado exactamente la misma mortalidad que los controles. No existiendo trabajo alguno sobre este tema en la literatura revisada, no nos es posible aportar una explicación que justifique el hallazgo. Cabría pensar que el efecto observado sobre la mortalidad no se deba al ácido folínico sino a algún elemento presente en el excipiente de la mezcla racémica utilizada (Lederlefolín ${ }^{\circledR}$ ). Aceptar o rechazar esta posibilidad requeriría nuevos trabajos.

No existen estudios que analicen el efecto del ácido folínico sobre la microcirculación en el intestino. En un trabajo publicado por Ulibarrena y cols. (18) se analiza el efecto del folínico sobre diversos parámetros hemodinámicos (macrocirculación). En concreto refiere que los animales tratados con Lederlefolin ${ }^{\circledR}$ mostraron una ligera mejoría de la tensión arterial media durante los primeros momentos de la reperfusión, sin que dicho efecto se mantuviera más allá de los quince minutos.

Sin embargo, en nuestro trabajo no hemos visto ningún efecto positivo del ácido folínico sobre la microcirculación de los tramos de intestino estudiados en ningún momento de la reperfusión. De hecho, no esperábamos que las modificaciones hemodinámicas de la macrocirculación tuvieran un reflejo paralelo a nivel capilar en el intestino, cuya regulación se ajusta a mecanismos y criterios diferentes.

En cambio, el tratamiento con levofolínico sí se ha seguido de una mejoría significativa de la perfusión capilar en el íleon terminal durante la reperfusión del intestino isquémico. Desconocemos si esta mejoría local se ha acompañado o no de una mejora en la hemodinámica de la macrocirculación.

En cuanto al daño mucoso, la experiencia llevada a cabo por Bilbao y cols. (15) con hembras de menor peso y 120 minutos de isquemia mostró un efecto positivo del folínico, ya que disminuyó el índice de daño mucoso desde 9,76 vs. 8,54 (p<0,05). En cambio, en nuestro mode- 
lo, este fármaco no ha demostrado capacidad de disminuir el daño de la mucosa intestinal tras la reperfusión. Esto pudiera deberse a que al ser el modelo menos agresivo desde el punto de vista local (ratas de mayor peso y menor tiempo de isquemia) (13) la experiencia se haya situado fuera de la "ventana terapéutica" del ácido folínico.

En cambio, el tratamiento con levofolínico ha tenido un efecto positivo a nivel de la mucosa, que se ha puesto de manifiesto en las valoraciones realizadas en el séptimo día de seguimiento. Este resultado es coherente con las experiencias previas de nuestro grupo que siempre han obtenido buenos resultados tras la administración de ácido folínico en modelos de isquemia intestinal.

Al analizar los resultados obtenidos en el estudio de los enzimas séricos en el grupo control, nuestros resultados son coherentes con lo publicado por otros autores. Así, en un modelo de 60 minutos de isquemia en conejo y tras una hora de reperfusión, Caglayan y cols. (12) observaron una duplicación de los valores de LDH y CK, mientras que la FA permanecía normal. El hecho de que en nuestra experiencia las concentraciones enzimáticas -respetando un patrón similar- resulten algo mayores puede deberse tanto a la diferencia de animal utilizado como al diferente tiempo de isquemia mesentérica.

Por otra parte, las mediciones realizadas por Thompson y cols. (19) en un modelo experimental diferente mostraron una elevación de la CK a las 4 horas de reperfusión y de la LDH y FA a las 24 horas. Nuestras mediciones a las 48 horas muestran valores normales para los tres enzimas.

El análisis del efecto de los fármacos sobre los niveles plasmáticos de estos enzimas permite apreciar una falta de correspondencia entre estas valoraciones y el resto de parámetros medidos en nuestra experiencia. De acuerdo con nuestra hipótesis inicial, el levofolínico debería aportar una protección más eficaz que el folínico, y por lo tanto acompañarse de niveles más bajos de enzimas séricos. En cambio, encontramos un retardo de al menos 24 horas en la normalización de los valores de LDH y un incremento significativo en la elevación inicial de la CK.

Con los datos disponibles no es posible ofrecer una explicación segura de este hecho. Es cierto que el levofolínico ha provocado una situación en algo diferente a la de los controles y animales tratados con folínico. Sin embargo, resulta difícil encontrar una explicación coherente del efecto observado. La mayor o más prolongada elevación de enzimas haría pensar en un agravamiento del daño; sin embargo, tanto nuestros estudios de daño mucoso como de microcirculación capilar demuestran un efecto positivo de la utilización del levofolínico en la prevención del daño. Quizás podrían conciliarse estos resultados contradictorios si el tratamiento con levofolínico estuviera transformando un cuadro intensamente agudo en otro subagudo de mejor pronóstico.

Por otra parte, hay muchos autores que opinan que los niveles aumentados de enzimas no aportan información relevante acerca de la extensión de la lesión intestinal ni de su reversibilidad $(15,19.20)$. Si ello fuera así, no debiera extrañar que la eficacia terapéutica de estos antioxidantes carezca de reflejo en los niveles enzimáticos.

En conclusión, el único punto en el que el levofolínico ha demostrado un efecto protector más intenso que el folínico ha sido en la lesión mucosa del íleon terminal, valorada en el séptimo día de evolución. En el resto de los parámetros las diferencias observadas no han alcanzado significación estadística. Por lo que se refiere al valor de las mediciones de flujo capilar durante la reperfusión del intestino isquémico, en este experimento han coincidido los valores más altos de flujo capilar en la reperfusión con un menor grado lesional de la mucosa, valorado en el séptimo día. Se requieren nuevos estudios con diferentes niveles de mortalidad y lesión anatomopatológica para establecer con claridad la "ventana lesional" en la que puede ser más útil la medición de microflujos para estudiar tratamientos del síndrome de reperfusión intestinal. Sin embargo, a la vista de nuestros resultados puede sostenerse que se trata de una herramienta interesante y cuyo uso en estos estudios está justificado.

\section{BIBLIOGRAFÍA}

1. McCord JM, Roy RS. Pathophysiology of superoxide: Roles in inflammation and ischemia. Canad J Physiol Pharmacol 1982; 60: 1346-51.

2. Kubes P, Ibbotson G, Russell J, Wallace JL, Granger DN. Role of platelet-activating factor in ischemia/reperfusion-induced leukocyte adherence. Am J Physiol 1990; 259: 300-5.

3. Carey C, Siegfried MR, Ma XL, Weyrich AS, Lefer AM. Antishock and endothelial protective actions of a NO donor in mesenteric ischemia and reperfusion. Circ Shock 1992; 38: 209-16.

4. Williams JP, Pechet TT, Weiser MR, Reid R, Kobzik L, Moore FD $\mathrm{Jr}$., et al. Intestinal reperfusion injury is mediated by IgM and complement. J Appl Physiol 1999; 86: 938-42.

5. Farber A, Connors JP, Friedlander RM, Wagner RJ, Powell RJ, Cronenwett JL. A specific inhibitor of apoptosis decreases tissue injury after intestinal ischemia-reperfusion in mice. J Vasc Surg 1999; 30: 752-60.

6. Rosario HS, Waldo SW, Becker SA, Schmid-Schonbein GW. Pancreatic trypsin increases matrix metalloproteinase-9 accumulation and activation during acute intestinal ischemia-reperfusion in the rat. Am J Pathol 2004; 164: 1707-16.

7. Granger DN, Benoit JN, Suzuki M, Grisham MB. Leukocyte adherence to venular endothelium during ischemia-reperfusion. Am J Physiol 1989; 257: 683-8.

8. Granger DN. Ischemia-reperfusion: mechanisms of microvascular dysfunction and the influence of risk factors for cardiovascular disease. Microcirculation 1999; 6: 167-78.

9. Goicoechea I, Fernández R, Otero B, Caramés J, Bilbao JE, GarcíaAlonso I. Pretreatment with antioxidants decreases damage due to reperfusion of isolated intestines in the abscense of leukocytes. Eur Surg Res 2003; 35: 293.

10. García-Alonso I, Ruiz de Gordejuela AG, Pérez-Agote J, Méndez JJ. Effects of antioxidant drugs on intestinal wall microflow in ischaemia-reperfusion syndrome. Eur Surg Res 2000; 32 (S1): 96. 
11. Park PO, Wallander J, Tufeson G, Haglund U. Cold ischemic and reperfusion injury in a model of small bowel transplantation in the rat. Eur Surg Res 1991; 23: 1-8.

12. Caglayan F, Caglayan O, Gunel E, Elcuman Y, Cakmak M. Intestinal ischemia-reperfusion and plasma enzyme levels. Pediatr Surg Int 2002; 18: 255-7.

13. Ortiz J, García-Alonso I, Portugal V, Méndez JJ. Influencia del peso corporal y del período de isquemia mesentérica aguda en la mortalidad de ratas sometidas a revascularización intestinal. Rev Esp Enferm Dig 1990; 77: 263-7.

14. Ulibarrena MA, García-Alonso I, Portugal V, García Redondo B, Méndez JJ. Monitorización hemodinámica en un modelo de shock por reperfusión intestinal en rata. Rev Esp Enferm Dig 1998; 90: 94-9.

15. Bilbao J, García-Alonso I, Portugal V, Barceló P, Apecechea A, Méndez JJ. Eficacia de tratamientos antioxidantes (ácido fólico y alfatocoferol) en las lesiones intestinales inducidas por reperfusión. Cir Esp 1992; 51: 262-5.
16. Bilbao J, García-Alonso I, Portugal V, Barceló P, Ortiz J, Méndez JJ. Utilidad terapéutica de fármacos antioxidantes en el síndrome de reperfusión intestinal experimental. Rev Esp Enferm Dig 1991; 80: $237-41$.

17. Ulibarrena MA, Conty JL, Emparan C, Portugal V, Bilbao J, GarcíaAlonso I. Acción de los inhibidores de los radicales libres del oxígeno sobre la mortalidad causada por el síndrome de isquemia-reperfusión intestinal. Rev Port Med Intensiva 1993; 2: 127.

18. Ulibarrena MA, Conty JL, Emparan C, Portugal V, Bilbao J, GarcíaAlonso I. Acción de los inhibidores de los radicales libres del oxígeno sobre la tensión arterial de la rata en el síndrome de isquemia-reperfusión intestinal. Rev Port Med Intensiva 1993; 2: 127.

19. Thompson JB, Bragg LE, West WW. Serum enzyme levels during intestinal ischemia. Ann Surg 1990; 211: 369-73.

20. Tanaka J, Malchesky PS, Omokawa S. Effects of prostaglandin I2, superoxide dismutase, and catalase on ischemia-reperfusion injury in liver transplantation. ASAIO Trans 1990; 36: 600-3. 